\title{
A SIMPLE ALTERNATIVE PROBLEM FOR FINDING PERIODIC SOLUTIONS OF SECOND ORDER ORDINARY DIFFERENTIAL SYSTEMS ${ }^{1}$
}

\author{
J. W. BEBERNES
}

ABSTRACT. Existence of solutions for $x^{\prime \prime}=f\left(t, x, x^{\prime}\right), x(0)=$ $x(1), x^{\prime}(0)=x^{\prime}(1)$ are proven by considering a simple alternative problem to which Leray-Schauder degree arguments can be directly applied.

1. Introduction. In this paper, we consider the existence of solutions to the periodic boundary value problem (PBVP)

$$
\begin{gathered}
x^{\prime \prime}=f\left(t, x, x^{\prime}\right), \\
x(0)=x(1), \quad x^{\prime}(0)=x^{\prime}(1) .
\end{gathered}
$$

Knobloch [4], Mawhin [5], Schmitt [6], and Bebernes and Schmitt [1] have recently considered this problem using degree-theoretic argumentseither finite or infinite dimensional.

Using only the basic properties of Leray-Schauder degree and applying these degree arguments to a simple alternative problem associated with (1)-(2), we obtain in this paper a single basic result (Theorem 2.1) which contains and in some cases permits slight generalizations of most of the results of the above mentioned papers.

2. The basic theorem. Let $I=[0,1], \boldsymbol{R}^{n}$ be $n$-dimensional Euclidean space with Euclidean norm $\|\cdot\|$ and inner product $\langle\cdot, \cdot\rangle$, and let $D \subset$ $I \times R^{n} \times R^{n}$ be a bounded open set in the relative topology of $I \times R^{n} \times R^{n}$ containing $\{(t, 0,0): t \in I\}$. Let $F: I \times \boldsymbol{R}^{n} \times \boldsymbol{R}^{n} \rightarrow \boldsymbol{R}^{n}$ be a continuous function and consider

$$
x^{\prime \prime}=F\left(t, x, x^{\prime}\right) .
$$

For each $\lambda \in[0,1]$, associate with (3) the equation

$$
x^{\prime \prime}=\lambda F\left(t, x, x^{\prime}\right)+(1-\lambda) x .
$$

Received by the editors April 6, 1973.

AMS (MOS) subject classifications (1970). Primary 34B15, 34C25; Secondary 47H15.

Key words and phrases. Periodic boundary value problems, alternative problems, Leray-Schauder degree, Nagumo-Hartman condition, Lyapunov-like functions.

1 This research was supported by the U.S. Air Force under Grant AFOSR-72-2379. 
and assume:

(H) If $x(t)$ is a solution of (4)-(2), then $\left(t, x(t), x^{\prime}(t)\right) \in D$ for all $t \in I$ or there exists $\tau \in I$ such that $\left(\tau, x(\tau), x^{\prime}(\tau)\right) \notin \bar{D}$.

THEOREM. 2.1. The periodic boundary value problem (3)-(2) has at least one solution such that $\left(t, x(t), x^{\prime}(t)\right) \in D$ for all $t \in I$.

PROOF. The periodic boundary value problem

$$
x^{\prime \prime}-x=0, \quad x(0)=x(1), \quad x^{\prime}(0)=x^{\prime}(1)
$$

has no nontrivial solutions. Let $H\left(t, x, x^{\prime}\right)=F\left(t, x, x^{\prime}\right)-x$, then $x(t)$ is a solution of (4)-(2) if and only if $x(t)$ is a solution of

$$
x(t)=\lambda \int_{0}^{1} G(t, s) H\left(s, x(s), x^{\prime}(s)\right) d s
$$

where $G(t, s)$ is the unique Green's function for (5).

Let $B=\left\{x \in C^{\prime}[0,1]: x(0)=x(1), x^{\prime}(0)=x^{\prime}(1)\right\}$ with norm

$$
|x|=\max _{I}\|x(t)\|+\max _{I}\left\|x^{\prime}(t)\right\|
$$

be the Banach space under consideration, and define

$$
\Omega=\left\{y \in B:\left(t, y(t), y^{\prime}(t)\right) \in D \text { for all } t \in I\right\} .
$$

Note that $\Omega$ is a bounded open subset of $B$.

Define the map $T: \bar{\Omega} \rightarrow B$ where $\Omega$ is the closure of $\Omega$ by

$$
(T y)(t)=\int_{0}^{1} G(t, s) H\left(s, y(s), y^{\prime}(s)\right) d s .
$$

By standard arguments, $T(\bar{\Omega}) \subset B, T$ is continuous, and $\operatorname{cl}(T(\bar{\Omega}))$ is compact in $B$.

If $0 \notin(I-\lambda T)(\partial \Omega)$ where $\partial \Omega$ is the boundary of $\Omega$ for all $\lambda \in[0,1]$, then by the invariance under compact homotopy property of the LeraySchauder degree [7, p. 92], the degree $\operatorname{deg}(I-\lambda T, \Omega, 0)=$ constant for all $\lambda \in[0,1]$. That $0 \in(I-\lambda T)(\partial \Omega)$ is equivalent to the existence of a solution $x(t)$ of the PBVP (4)-(2) with $\left(t, x(t), x^{\prime}(t)\right) \in \bar{D}$ for all $t \in I$ and $\left(t, x(t), x^{\prime}(t)\right) \in \partial D$ for some $t \in I$; but by assumption $(\mathrm{H})$ there exists no such solution $x(t)$ of (4)-(2) with $\left(t, x(t), x^{\prime}(t)\right) \in \bar{D}$ for all $t \in I$ and $\left(t, x(t), x^{\prime}(t)\right) \in \partial \bar{D}$ for some $t \in I$. Hence, $\operatorname{deg}(I-T, \Omega, 0)=\operatorname{deg}(I, \Omega, 0)=$ 1. By the existence property of the Leray-Schauder degree [7, p. 88], there exists $x \in \Omega$ such that $(I-T) x=0$. This means that there exists a solution $x(t)$ of the PBVP (3)-(2) with $\left(t, x(t), x^{\prime}(t)\right) \in D$ for all $t \in I$.

3. Applications of the basic theorem. In this section, we illustrate how Theorem 2.1 can be used to prove existence results for PBVP (1)-(2). 
The first result is known (e.g., [1], [4], or [5]), but it well illustrates the power of our basic theorem.

THEOREM 3.1. If $f\left(t, x, x^{\prime}\right)$ is continuous on $E_{R}=\left\{\left(t, x, x^{\prime}\right): t \in I\right.$, $\left.\|x\|<R,\left\|x^{\prime}\right\|<\infty\right\}$ and satisfies:

(8) $\left\|x^{\prime}\right\|^{2}+\left\langle x, f\left(t, x, x^{\prime}\right)\right\rangle>0$ for all $\left(t, x, x^{\prime}\right) \in E_{R}$ provided $\|x\|=R$ and $\left\langle x, x^{\prime}\right\rangle=0$;

(9) $\left\|f\left(t, x, x^{\prime}\right)\right\| \leqq \varphi\left(\left\|x^{\prime}\right\|\right)$ for all $\left(t, x, x^{\prime}\right) \in E_{R}$ where $\varphi$ is a positive continuous function on $[0, \infty)$ with $\int^{\infty} s / \varphi(s) d s=+\infty$;

(10) there exists $\alpha \geqq 0, K \geqq 0$ such that

$$
\left\|f\left(t, x, x^{\prime}\right)\right\| \leqq 2 \alpha\left(\left\|x^{\prime}\right\|^{2}+\left\langle x, f\left(t, x, x^{\prime}\right)\right\rangle\right)+K \text { for all }\left(t, x, x^{\prime}\right) \in E_{R} ;
$$

then there exists a solution $x(t)$ of the PBVP (1)-(2) with $\left(t, x(t), x^{\prime}(t)\right) \in E_{R}$.

Proof. Let $\delta_{M}(s)$ be a continuous function on $[0, \infty)$ with $\delta_{M}(s)=1$ on $[0, M]$ and $\delta_{M}(s)=0$ for $s \geqq 2 M$ where $M=M(\alpha, K, R)$ is the NagumoHartman bound (see Hartman [3, p. 429]).

Define

$$
\begin{array}{ll}
F\left(t, x, x^{\prime}\right)=\delta_{M}\left(\left\|x^{\prime}\right\|\right) f\left(t, x, x^{\prime}\right) & \text { on } E_{R}, \text { and } \\
F\left(t, x, x^{\prime}\right)=(R /\|x\|) F\left(t, R x /\|x\|, x^{\prime}\right) & \text { if }\|x\| \geqq R .
\end{array}
$$

Then $F\left(t, x, x^{\prime}\right)$ is continuous and bounded on $I \times R^{n} \times R^{n}$ and satisfies (8) provided $\|x\| \geqq R$ and $\left\langle x, x^{\prime}\right\rangle=0$, (9), and (10) for all $\left(t, x, x^{\prime}\right) \in$ $I \times R^{n} \times R^{n}$.

The proof will be completed by showing that there can be constructed an open bounded set $D \subset I \times R^{n} \times R^{n}$ containing $\{(t, 0,0): t \in I\}$ such that solutions of PBVP (4)-(2) satisfy hypothesis (H) relative to $D$.

For each $\lambda \in[0,1]$, let $F_{\lambda}\left(t, x, x^{\prime}\right)=\lambda F\left(t, x, x^{\prime}\right)+(1-\lambda) F\left(t, x, x^{\prime}\right)$ where $F$ is defined as above. Then for all $\lambda \in[0,1]$ and all $\left(t, x, x^{\prime}\right) \in$ $I \times \boldsymbol{R}^{n} \times \boldsymbol{R}^{n}$,

$$
\left\|x^{\prime}\right\|^{2}+\left\langle x, F_{\lambda}\left(t, x, x^{\prime}\right)\right\rangle>0 \text { provided }\|x\| \geqq R
$$

and $\left\langle x, x^{\prime}\right\rangle=0$. Let $x(t)$ be any solution of (4)-(2). Define $u(t)=\|x(t)\|^{2}=$ $\langle x(t), x(t)\rangle$. Because $u(t)$ satisfies the periodic boundary conditions (2), $u(t)$ can assume its maximum at $t_{0} \in I$ only if $u\left(t_{0}\right)=0, u^{\prime}\left(t_{0}\right) \leqq 0$. Claim $u(t)<R^{2}$ for all $t \in I$. Assume not; then there exists $t_{0} \in I$ at which $u(t)$ assumes its maximum with $u\left(t_{0}\right) \geqq R^{2}, u^{\prime}\left(t_{0}\right)=0$, and $u^{\prime \prime}\left(t_{0}\right) \leqq 0$. But (11) implies that $u^{\prime \prime}\left(t_{0}\right)>0$ which is a contradiction. Hence, $\|x(t)\|<R$ for all $t \in I$. For $\left(t, x, x^{\prime}\right) \in E_{R}$ and $\lambda \in[0,1], F_{\lambda}\left(t, x, x^{\prime}\right)$ is bounded which implies that $F_{\lambda}\left(t, x, x^{\prime}\right)$ satisfies a Nagumo-Hartman condition (conditions (9) and (10) with $\alpha=0$ and a $K^{\prime}$ in general different from $K$ and $\varphi(s)=K^{\prime}$ ). Hence, there exists an $M^{\prime}>0$ such that if $x(t)$ is any solution of (4) on $I$ with $\|x(t)\|<R$, then $\left\|x^{\prime}(t)\right\|<M^{\prime}$. 
Define $D=\left\{\left(t, x, x^{\prime}\right): t \in I,\|x\|<R,\left\|x^{\prime}\right\|<M^{\prime}\right\}$. From the observations made above it is immediate that solutions of (4)-(2) satisfy $(H)$ relative to $D$. By Theorem 2.1, the PBVP (3)-(2) has a solution $x(t)$ with $\|x(t)\|<R$. Since $F\left(t, x, x^{\prime}\right)$ satisfies (9) and (10), $\left\|x^{\prime}(t)\right\|<M$ on $[0,1]$ which implies that $x(t)$ is a solution of PBVP (1)-(2) on $I$ with $\left(t, x(t), x^{\prime}(t)\right) \in E_{R}$.

Equality can be permitted in (8) by an approximating argument like the one given in $[3$, p. 433].

The preceding theorem can be generalized by replacing $\|x\|^{2}$ by a function $V(t, x)$ which plays essentially the same role. In so doing, we obtain results similar to those obtained by Knobloch [4] and Mawhin [5].

Assume $f\left(t, x, x^{\prime}\right): I \times \boldsymbol{R}^{n} \times \boldsymbol{R}^{n} \rightarrow \boldsymbol{R}^{n}$ is continuous and let $\boldsymbol{R}^{+}$denote the nonnegative reals.

Definition. Let $V \in C^{2}\left(I \times R^{n} \times R^{n}, R^{+}\right)$be such that:

(a) there exists $R>0$ such that $\Phi \equiv\left\{x \in R^{n}: V(t, x)<R, t \in I\right\}$ is bounded,

(b) $U\left(t, x, x^{\prime}\right) \equiv V_{t t}(t, x)+2\left\langle V_{t x}(t, x), x^{\prime}\right\rangle+\left\langle V_{x x}(t, x) x^{\prime}, x^{\prime}\right\rangle \geqq 0$,

(c) $V_{f}^{\prime \prime}(t, x)=U\left(t, x, x^{\prime}\right)+\left\langle V_{x}(t, x), f\left(t, x, x^{\prime}\right)\right\rangle>0$ provided $V(t, x)=R$ and $V_{t}(t, x)+\left\langle V_{x}(t, x), x^{\prime}\right\rangle=0$,

(d) $\left\langle V_{x}(t, x), x\right\rangle>0$ for all $(t, x)$ such that $V(t, x)=R$,

(e) $V(0, x)=V(1, x), V_{t}(0, x)+\left\langle V_{x}(0, x), x^{\prime}\right\rangle \geqq V_{t}(1, x)+\left\langle V_{x}(1, x), x^{\prime}\right\rangle$.

Any such $V$ is called a bounding Lyapunov function relative to (1).

THEOREM 3.2. If $V$ is a bounding Lyapunov function for (1), then for every $\lambda \in[0,1]$ every solution $x(t)$ of the PBVP:

$$
x^{\prime \prime}=f_{\lambda}\left(t, x, x^{\prime}\right)
$$

where $f_{\lambda}=\lambda f+(1-\lambda) f$ is such that $V(\tau, x(\tau))>R$ for some $\tau \in I$ or $V(t, x(t))<R$ for all $t \in I$.

PROOF. Let $x(t)$ be any solution of the PBVP (12)-(2) and let $m(t)=$ $V(t, x(t))$, then $m^{\prime}(t)=V_{t}(t, x(t))+\left\langle V_{x}(t, x(t)), x^{\prime}(t)\right\rangle$ and

$$
m^{\prime \prime}(t)=U\left(t, x(t), x^{\prime}(t)\right)+\left\langle V_{x}(t, x(t)), f_{\lambda}\left(t, x(t), x^{\prime}(t)\right)\right\rangle .
$$

By (b), (c), and (d), $m^{\prime \prime}(t)>0$ if $V(t, x(t))=R$ and $V_{t}(t, x(t))+$ $\left\langle V_{x}(t, x(t)), x^{\prime}(t)\right\rangle=0$. If there exists $\tau \in I$ such that $m(\tau)>R$, we are through.

Assume $m(t) \leqq R$ for all $t \in[0,1]$. If there exists $t_{0} \in I$ such that $m\left(t_{0}\right)=R$, then $m^{\prime}\left(t_{0}\right)=0$ and $m^{\prime \prime}\left(t_{0}\right) \leqq 0$ since $m(0)=m(1)$ and $m^{\prime}(0) \geqq m^{\prime}(1)$ by $(\mathrm{e})$. But this is impossible by the observation made above that $m^{\prime \prime}\left(t_{0}\right)>0$. Hence, $m(t)<R$ on $I$ and the conclusion of the theorem follows.

Our next theorem is similar to Theorem 6.1 [5]. 
THEOREM 3.3. If $V$ is a positive definite bounding Lyapunov function relative to (1) and if there exists $S>0$ such that for any $\lambda \in[0,1]$ any solution $x(t)$ of $P B V P(12)-(2)$ with $V(t, x(t))<R$ on I satisfies $\left\|x^{\prime}(t)\right\|<S$ for $t \in I$, then PBVP (1)-(2) has at least one solution $x(t)$ with $V(t, x(t))<R$.

Proof. Let $D=\left\{\left(t, x, x^{\prime}\right): t \in I, V(t, x)<R,\left\|x^{\prime}\right\|<S\right\}$. By Theorem 3.2 , solutions of (12)-(2) satisfy $(H)$ relative to $D$. Hence, by Theorem 1.2 , the conclusion follows.

There are several ways of ensuring the a priori bound condition on the derivative of solutions of (12)-(2) and hence we have the following corollaries.

COROLLARY 3.4. If $V$ is a bounding positive definite Lyapunov function for (1) and iff $\left(t, x, x^{\prime}\right)$ satisfies (9) and (10) for all $t \in I, x \in \Phi,\left\|x^{\prime}\right\|<\infty$, then PBVP (1)-(2) has a solution $x(t) \in \Phi$ for all $t \in I$.

COROLlaRY 3.5. If $V$ is a bounding positive definite Lyapunov function for (1), if $f\left(t, x, x^{\prime}\right)$ satisfies (9) for all $t \in I, x \in \Phi,\left\|x^{\prime}\right\|<\infty$, and if there exists $\beta \geqq 0, L \geqq 0$ such that

$$
\left\|f\left(t, x, x^{\prime}\right)\right\| \leqq \beta\left(U\left(t, x, x^{\prime}\right)+\left\langle V_{x}(t, x), f\left(t, x, x^{\prime}\right)\right\rangle\right)+L
$$

for all $t \in I, x \in \Phi$, and $\left\|x^{\prime}\right\| \leqq \infty$, then $P B V P(1)-(2)$ has a solution $x(t) \in \Phi$ for all $t \in I$.

COROLlaRY 3.6. If $V$ is a bounding positive definite Lyapunov function for (1), if $f\left(t, x, x^{\prime}\right)$ satisfies (9) for all $t \in I, x \in \Phi,\left\|x^{\prime}\right\|<\infty$, and if there exists a function $\rho(t) \in C^{2}(I)$ such that

$$
\left\|f\left(t, x, x^{\prime}\right)\right\| \leqq \rho^{\prime \prime}(t) \text { for all } t \in I, x \in \Phi,\left\|x^{\prime}\right\|<\infty,
$$

then PBVP (1)-(2) has a solution $x(t) \in \Phi$ for all $t \in I$.

4. Further consequences. In this section, we present two further applications of Theorem 2.1. The first theorem presented shows that the bounding set $\Phi$ need not be given in terms of a bounding Lyapunov function. Assume $f\left(t, x, x^{\prime}\right): I \times \boldsymbol{R}^{n} \times \boldsymbol{R}^{n} \rightarrow \boldsymbol{R}^{n}$ is continuous.

THEOREM 4.1. Let $G$ be a bounded convex open set in $\boldsymbol{R}^{n}$ containing 0 and assume there is a function $N: \partial G \rightarrow \boldsymbol{R}^{n}$ satisfying:

$$
\begin{aligned}
& \langle N(x), x\rangle>0 \text { for all } x \in \partial G, \\
& \text { (17) } G \subseteq\{y:\langle N(x), y-x\rangle \leqq 0 \text { for each } x \in \partial G\} \text {, } \\
& \left\langle N(x), f\left(t, x, x^{\prime}\right)\right\rangle>0 \text { for all } t \in I, x \in \partial G, \\
& x^{\prime} \text { with }\left\langle N(x), x^{\prime}\right\rangle=0,
\end{aligned}
$$

then for every $\lambda \in[0,1]$ every solution $x(t)$ of $(12)-(2)$ is such that $x(\tau) \notin G$ for some $\tau \in I$ or $x(t) \in G$ for all $t \in I$. 
REMARK. Conditions (16) and (17) say that $N(x)$ is an outer normal for G. Gustafson and Schmitt [2] have used a similar outer normal condition to study existence of periodic solutions for delay differential equations.

PROOF. Let $x(t)$ be any solution of (12)-(2). If $x(\tau) \notin G$ for some $\tau \in I$, we are through so assume $x(t) \in G$ for all $t \in I$.

If $x\left(t_{0}\right) \in \partial G$ for some $t_{0} \in I$, we may assume $t_{0} \in[0,1)$. By (16) and (18), $\left\langle N\left(x\left(t_{0}\right)\right), f_{\lambda}\left(t_{0}, x\left(t_{0}\right), x^{\prime}\left(t_{0}\right)\right)\right\rangle>0$ and hence there is an $h>0$ such that $\left\langle N\left(x\left(t_{0}\right)\right), x^{\prime \prime}(t)\right\rangle>0$ for all $t \in\left[t_{0}, t_{0}+h\right)$. Since $x(t) \in G,\left\langle N\left(x\left(t_{0}\right)\right), x^{\prime}\left(t_{0}\right)\right\rangle=$ 0 . Looking at the Taylor expansion for $x(t)$, we have immediately that

$$
\begin{aligned}
\left\langle N\left(x\left(t_{0}\right)\right),\right. & \left.x(t)-x\left(t_{0}\right)\right\rangle \\
& =\left(t-t_{0}\right)\left\langle N\left(x\left(t_{0}\right)\right), x^{\prime}\left(t_{0}\right)\right\rangle+\frac{1}{2}\left(t-t_{0}\right)^{2}\left\langle N\left(x\left(t_{0}\right)\right), y(\bar{\xi})\right\rangle
\end{aligned}
$$

where $y(\bar{\xi})=\left(x_{1}^{\prime \prime}(\xi), \cdots, x_{n}^{\prime \prime}\left(\xi_{n}\right)\right)$ and $t_{0}<\xi_{i}<t<t_{0}+h$ for all $i=1, \cdots, n$. From this, $\left\langle N\left(x\left(t_{0}\right)\right), x(t)-x\left(t_{0}\right)\right\rangle>0$ meaning that $x(t) \notin G$, which is a contradiction.

Our existence theorem then follows.

THEOREM 4.2. If $G$ is a bounded convex open set in $\boldsymbol{R}^{n}$ containing 0 , if there is a function $N: \partial G \rightarrow R^{n}$ satisfying (16), (17), and (18), and if there exists $S>0$ such that for any $\lambda \in[0,1]$ any solution $x(t)$ of $P B V P(12)-(2)$ with $x(t) \in G$ for all $t \in I$ satisfies $\left\|x^{\prime}(t)\right\|<S$ for $t \in I$, then $P B V P$ (1)-(2) has at least one solution with $x(t) \in G$ for all $t \in I$.

Proof. Let $D=\left\{\left(t, x, x^{\prime}\right): t \in I, x \in G,\left\|x^{\prime}\right\|<S\right\}$. By Theorem 4.1 solutions of (11)-(2) satisfy $(\mathrm{H})$ relative to $D$. Result then follows immediately from Theorem 2.1 .

REMARK. One can state corollaries of the above theorem analogous to Corollaries 3.4, 3.5, and 3.6.

In $R^{n}$, let $x \leqq y$ if and only if $x_{i} \leqq y_{i}, 1 \leqq i \leqq n$, and $x<y$ if and only if $x_{i}<y_{i}, 1 \leqq i \leqq n$.

Let $f\left(t, x, x^{\prime}\right)$ be continuous on $\left\{\left(t, x, x^{\prime}\right): t \in I, \alpha(t) \leqq x \leqq \beta(t), x^{\prime} \in R^{n}\right\}$ where $\alpha, \beta: I \rightarrow \boldsymbol{R}^{n}, \alpha(t)<0<\beta(t)$ are twice continuously differentiable with

$$
\alpha(0)=\alpha(1), \quad \beta(0)=\beta(1), \quad \alpha^{\prime}(0) \geqq \alpha^{\prime}(1), \quad \beta^{\prime}(0) \leqq \beta^{\prime}(1) .
$$

Assume also that $\alpha, \beta$ are strict lower, upper solutions of (1), i.e.,

$$
\begin{aligned}
& \alpha^{\prime \prime}(t)>f_{i}\left(t, x_{1}, \cdots, x_{i-1}, \alpha_{i}(t), x_{i+1}, \cdots, x_{n}, x_{1}^{\prime}, \cdots,\right. \\
& \left.x_{i-1}^{\prime}, \alpha_{i}^{\prime}(t), x_{i+1}^{\prime}, \cdots, x_{n}^{\prime}\right), \\
& \beta_{i}^{\prime \prime}(t)<f_{i}\left(t, x_{1}, \cdots, x_{i-1}, \beta_{i}(t), x_{i+1}, \cdots, x_{n}, x_{1}^{\prime}, \cdots,\right. \\
& \left.x_{i-1}^{\prime}, \beta_{i}^{\prime}(t), x_{i+1}^{\prime}, \cdots, x_{n}^{\prime}\right),
\end{aligned}
$$


and

$$
\alpha_{i}^{\prime \prime}(t)>\alpha_{i}(t), \quad \beta_{i}^{\prime \prime}(t)<\beta_{i}(t)
$$

for $\alpha_{j}(t) \leqq x_{j} \leqq \beta_{j}(t), j \neq i, i=1, \cdots, n$.

We now can state our final result.

THEOREM 4.3. If $f$ is continuous on $\left\{\left(t, x, x^{\prime}\right): t \in I, \alpha(t) \leqq x \leqq \beta(t)\right.$, $\left.x^{\prime} \in \boldsymbol{R}^{n}\right\}$ where $\alpha, \beta$ are strict periodic lower, upper solutions of (1) satisfying (19), (20), and (21), and if there exists $S>0$ such that for any $\lambda \in[0,1]$ any solution $x(t)$ of $(12)-(2)$ with $\alpha(t) \leqq x(t) \leqq \beta(t)$ on I satisfies $\left\|x^{\prime}(t)\right\|<S$ then PBVP (1)-(2) has a solution $x(t)$ with $\alpha(t)<x(t)<\beta(t)$.

The proof is similar to those previously given and is for this reason omitted. By a proper modification of $f\left(t, x, x^{\prime}\right)$, condition (21) can be dropped and equality can be permitted in (20). With that observation, we have a generalization of Theorem 4.1 in [1].

\section{REFERENCES}

1. J. Bebernes and K. Schmitt, Periodic boundary value problems for syste.is of second order differential equations, J. Differential Equations 13 (1973), 32-47.

2. G. Gustafson and K. Schmitt, $A$ note on periodic solutions for delay-differential systems, J. Differential Equations 13 (1973), 567-587.

3. P. Hartman, Ordinary differential equations, Wiley, New York, 1964. MR 30 $\# 1270$.

4. $\mathrm{H}$. Knobloch, On the existence of periodic solutions of second order vector differential equations, J. Differential Equations 9 (1971), 67-85. MR 43 \#3557.

5. J. Mawhin, Boundary value problems for nonlinear second order vector differential equations (to appear).

6. K. Schmitt, Periodic solutions of systems of second order differential equations, J. Differential Equations 11 (1972), 180-192. MR 45 \#3858.

7. J. Schwartz, Nonlinear functional analysis, Gordon and Breach, New York, 1969.

Department of Mathematics, University of Colorado, Boulder, Colorado 80302 